\title{
Perturbation theorems for local integrated semigroups and their applications
}

\author{
by \\ Sheng Wang Wang, Mei Ying Wang and Yan Shen (Nanjing)
}

\begin{abstract}
Motivated by a great deal of interest in operators that may not be densely defined and do not generate global integrated semigroups, we establish general perturbation theorems for local integrated semigroups and describe their applications to local complete second order abstract differential equations.
\end{abstract}

1. Introduction. Let $X$ be a complex Banach space, and consider the following local complete second order abstract differential equation on $X$ :

$$
u^{\prime \prime}(t)=A u(t)+B u^{\prime}(t), \quad t \in[0, \tau) ; \quad u(0)=x, \quad u^{\prime}(0)=y,
$$

where $0<\tau \leq \infty$ is fixed, and $A$ and $B$ are closed linear operators on $X$ which are not necessarily densely defined. As shown in [8, Chapter VIII] equation (1.1) may have solutions which are either not exponentially bounded or not defined on $[0, \infty)$ for dense sets of initial data. We deal with these two cases, in this paper, from the following point of view: we first establish several perturbation theorems for local integrated semigroups in Sections 2 and 3; then, in Section 4, we reduce (1.1) to two kinds of systems of first order differential equations and apply the results obtained in Sections 2 and 3 to prove the wellposedness of these two systems in a certain sense.

The methods employed in this paper seem to be natural and direct, and the results obtained are more general than the perturbation theorems for strongly continuous semigroups and integrated semigroups appearing in $[6$, $7,9,10,16]$. In fact, to reach our target, we follow a quite different path from these references.

As regards the theory of strongly continuous semigroups, cosine operator families and their applications to partial differential equations, the reader will find the books $[9,12]$ by J. Goldstein and A. Pazy, respectively, to be of importance. As regards the theory of integrated semigroups and regularized

2000 Mathematics Subject Classification: Primary 47D06; Secondary 47B40. 
semigroups and their applications to partial differential equations, the book [4] by R. deLaubenfels is of importance.

Throughout, $L(X)$ is the algebra of all bounded linear operators on $X$. For a closed linear operator $A$ on $X, D(A)$ and $\operatorname{Im}(A)$ denote its domain and image, respectively, while $\varrho(A)$ and $\sigma(A)$ stand for the resolvent set and spectrum of $A$. For $\lambda \in \varrho(A)$, set $R(\lambda, A)=(\lambda-A)^{-1}$. The set $D(A)$, equipped with the graph norm, is denoted by $[D(A)]$ and is a Banach space. If $B \in L(X)$ satisfies: $B x \in D(A)$ and $B A x=A B x$ for all $x \in D(A)$ then we write $B A \subseteq A B$ and say that $A$ and $B$ commute.

Definition 1.1. Let $n \in \mathbb{N}$ and $0<\tau \leq \infty$ be given. A strongly continuous family of operators $\{S(t)\}_{t \in[0, \tau)} \subset L(X)$ is a local n-times integrated semigroup if:

(i) $S(0)=0$.

(ii) For every $x \in X$, one has

$$
S(t) S(s) x=\frac{1}{(n-1) !}\left[\int_{t}^{s+t}-\int_{0}^{s}\right](s+t-r)^{n-1} S(r) x d r
$$

whenever $0 \leq s, t, s+t<\tau$.

The $n$-times integrated semigroup $\{S(t)\}_{t \in[0, \tau)}$ is nondegenerate if $S(t) x$ $=0$ for all $t \in[0, \tau)$ implies that $x=0$. The generator of a nondegenerate $\{S(t)\}_{t \in[0, \tau)}$ is the following operator:

$$
\begin{aligned}
& D(A):=\{x \in X: \exists y \in X \text { such that } \\
& \left.\qquad S(t) x=\int_{0}^{t} S(r) y d r+\frac{t^{n}}{n !} x \forall t \in[0, \tau)\right\} ; \\
& A x:=y \quad \forall x \in D(A) .
\end{aligned}
$$

The generator $A$ is well defined by nondegeneracy.

Throughout, the word "local" will be omitted from all our statements for simplicity.

Definition 1.2. A function $u(\cdot, x):[0, \tau) \rightarrow X$ is a strong solution of the abstract differential equation

$$
u^{\prime}(t)=A u(t), \quad t \in[0, \tau) ; \quad u(0)=x, \quad x \in X,
$$

if $u(\cdot, x) \in C^{1}([0, \tau), X) \cap C([0, \tau),[D(A)])$ and satisfies (1.2).

Let $n \in \mathbb{N}$. A function $v(\cdot, x):[0, \tau) \rightarrow X$ is an $n$-times integrated mild solution of $(1.2)$ if $v(0, x)=0, v(\cdot, x) \in C([0, \tau), X), \int_{0}^{t} v(s, x) d s \in D(A)$ 
and

$$
v(t, x)=A \int_{0}^{t} v(s, x) d s+\frac{t^{n}}{n !} x, \quad x \in X, t \in[0, \tau) .
$$

In [2], (1.3) is written as $C_{n+1}(\tau)$ and if for every $x \in X,(1.3)$ has a unique solution then $C_{n+1}(\tau)$ is said to be well-posed (see [2, Section 1]).

Some basic properties of integrated semigroups stated in the following theorem and proposition can be found in [5] for the global case. But all these properties remain valid for our local case (see also [2]).

TheOREM 1.3. Assume $A$ is a closed linear operator on $X$ and $\{S(t)\}_{t \in[0, \tau)}$ $\subset L(X)$ is a strongly continuous family of bounded linear operators. Then the following conditions (i)-(iii) are equivalent:

(i) $\{S(t)\}_{t \in[0, \tau)}$ is an n-times integrated semigroup generated by $A$.

(ii) (a) For every $x \in X$ and $t \in[0, \tau), \int_{0}^{t} S(r) x d r \in D(A)$ and

$$
A \int_{0}^{t} S(r) x d r=S(t) x-\frac{t^{n}}{n !} x .
$$

(b) Either $S(t) A \subseteq A S(t)$ or the solutions of (1.2) are unique.

(iii) (1.2) has a unique $n$-times integrated mild solution for every $x \in X$. If the equivalent conditions (i)-(iii) hold, then

(iv) (1.2) has a unique strong solution for every $x \in D\left(A^{n+1}\right)$ given by $u(t, x):=S^{(n)}(t) x$.

The fact that $u(t, x)=S^{(n)}(t) x$ is the unique strong solution of (1.2) for $x \in D\left(A^{n+1}\right)$ is stated in

Proposition 1.4. If $\{S(t)\}_{t \in[0, \tau)}$ is an n-times integrated semigroup generated by $A$ then:

(i) For $k \in \mathbb{N}$ and $x \in D\left(A^{k}\right)$, we have $S^{(k-1)}(t) x \in D(A)(t \in[0, \tau))$ and

$$
\begin{aligned}
& A S^{(k-1)}(t) x=S^{(k)}(t) x-\frac{t^{n-k}}{(n-k) !} x \quad \forall t \in[0, \tau), 1 \leq k \leq n, \\
& A S^{(k-1)}(t) x=S^{(k)}(t) x \quad \forall t \in[0, \tau), \quad k>n .
\end{aligned}
$$

The first relation, together with $S(0)=0$, implies $S^{(k)}(0) x=0$ for all $x \in D\left(A^{k}\right), 1 \leq k \leq n-1$, and $S^{(n)}(0) x=x$ for $x \in D\left(A^{n}\right)$.

(ii) $\varrho(A)$ is nonempty and there exist $\alpha, \beta>0$ such that ([2])

$$
E(\alpha, \beta):=\left\{\lambda \in \mathbb{C}: \operatorname{Re} \lambda \geq \beta,|\operatorname{Im} \lambda| \leq e^{\alpha \operatorname{Re} \lambda}\right\} \subseteq \varrho(A) .
$$

(iii) Every operator $B \in L(X)$ commuting with $A$ also commutes with $S(t)$ for all $t \in[0, \tau)$. 
Proof. We only have to show (iii). [2, Proposition 3.1(c)] gives $S(t) A \subseteq$ $A S(t)$. Let $x \in X$. From

$$
\begin{aligned}
& A \int_{0}^{t} S(s) B x d s=S(t) B x-\frac{t^{n}}{n !} B x \\
& A \int_{0}^{t} B S(s) x d s=B A \int_{0}^{t} S(s) x d s=B S(t) x-\frac{t^{n}}{n !} B x
\end{aligned}
$$

and the uniqueness of solutions of (1.2), it follows that $S(t) B=B S(t)$.

We now define the concept and state some basic properties of $n$-times integrated cosine operator families.

Definition 1.5. Let $n \in \mathbb{N}$ and $0<\tau \leq \infty$ be fixed. A strongly continuous family of operators $\{\mathcal{C}(t)\}_{t \in[0, \tau)} \subset L(X)$ is an n-times integrated cosine operator family if:

(i) $\mathcal{C}(0)=0$.

(ii) For every $x \in X$, we have

$$
\begin{aligned}
2 \mathcal{C}(s) \mathcal{C}(t) x= & \frac{1}{(n-1) !}\left\{(-1)^{n} \int_{0}^{|s-t|}(|s-t|-r)^{n-1} \mathcal{C}(r) x d r\right. \\
& +\left[\int_{0}^{s+t}-\int_{0}^{s}-\int_{0}^{t}\right](s+t-r)^{n-1} \mathcal{C}(r) x d r \\
& \left.+\int_{0}^{t}(s-t+r)^{n-1} \mathcal{C}(r) x d r+\int_{0}^{s}(t-s+r)^{n-1} \mathcal{C}(r) x d r\right\}
\end{aligned}
$$

whenever $0 \leq s, t, s+t<\tau$.

$\{\mathcal{C}(t)\}_{t \in[0, \tau)}$ is nondegenerate if $\mathcal{C}(t) x=0$ for all $t \in[0, \tau)$ implies $x=0$. The generator of a nondegenerate $\{\mathcal{C}(t)\}_{t \in[0, \tau)}$ is the following operator:

$$
\begin{gathered}
D(A):=\{x \in X: \exists y \in X \text { such that } \\
\left.\qquad \int_{0}^{t}(t-r) \mathcal{C}(r) y d r=\mathcal{C}(t) x-\frac{t^{n}}{n !} x \forall t \in[0, \tau)\right\} ; \\
A x:=y \quad \forall x \in D(A) .
\end{gathered}
$$

It is also easy to see that $A$ is well defined by nondegeneracy.

Definition 1.6. A function $u(\cdot, x, y):[0, \tau) \rightarrow X$ is a strong solution of the second order abstract differential equation

$$
u^{\prime \prime}(t)=A u(t), \quad t \in[0, \tau) ; \quad u(0)=x, \quad u^{\prime}(0)=y, \quad x, y \in X,
$$
if $u(\cdot, x, y) \in C^{2}([0, \tau), X) \cap C([0, \tau),[D(A)])$ and satisfies (1.4). 
Let $n \in \mathbb{N}$. A function $v(\cdot, x, y):[0, \tau) \rightarrow X$ is an $n$-times integrated mild solution of (1.4) if $v(0, x, y)=0, v(\cdot, x, y) \in C([0, \tau), X), \int_{0}^{t}(t-s) v(s, x, y) d s$ $\in D(A)$ for $t \in[0, \tau)$ and

$$
\begin{aligned}
v(t, x, y)= & A \int_{0}^{t}(t-s) v(s, x, y) d s \\
& +\frac{t^{n}}{n !} x+\frac{t^{n+1}}{(n+1) !} y, \quad x, y \in X, t \in[0, \tau) .
\end{aligned}
$$

As usual, for a nonnegative real number $\alpha$ we denote by $[\alpha]$ the greatest nonnegative integer not greater than $\alpha$.

TheOREm 1.7. Assume $A$ is a closed linear operator on $X$ and $\{\mathcal{C}(t)\}_{t \in[0, \tau)}$ $\subset L(X)$ is a strongly continuous family of operators. Then the following conditions (i)-(iii) are equivalent:

(i) $\{\mathcal{C}(t)\}_{t \in[0, \tau)}$ is an n-times integrated cosine operator family generated by $A$.

(a) $\mathcal{C}(t) A \subseteq A \mathcal{C}(t)$.

(b) For $x \in X$ and $t \in[0, \tau), \int_{0}^{t}(t-r) \mathcal{C}(r) x d r \in D(A)$ and

$$
A \int_{0}^{t}(t-r) \mathcal{C}(r) x d r=\mathcal{C}(t) x-\frac{t^{n}}{n !} x .
$$

(iii) (1.4) has a unique $n$-times integrated mild solution for every pair $x, y \in X$.

If the equivalent conditions (i)-(iii) hold, then

(iv) (1.4) has a unique strong solution for all $x, y \in D\left(A^{1+[(n+1) / 2]}\right)$.

To prove the theorem we need the following lemma.

Lemma 1.8. If A satisfies (ii) of Theorem 1.7 then there exists $\omega_{0}>0$ such that $\left(\omega_{0}^{2}, \infty\right) \subseteq \varrho(A)$.

Proof. Define

$$
R_{\lambda}(t) x:=\int_{0}^{t} e^{-\lambda s}\left[\int_{0}^{s} \mathcal{C}(r) x d r\right] d s \quad \forall x \in X, t \in(0, \tau) \text { and } \lambda>0 .
$$

Apply $A$ to both sides and use integration by parts twice to find

$$
\begin{aligned}
A R_{\lambda}(t) x & =A \int_{0}^{t} e^{-\lambda s} d \int_{0}^{s}(s-r) \mathcal{C}(r) x d r \\
& =e^{-\lambda t}\left[\mathcal{C}(t) x-\frac{t^{n}}{n !} x\right]+\lambda \int_{0}^{t} e^{-\lambda s}\left[\mathcal{C}(s) x-\frac{s^{n}}{n !} x\right] d s
\end{aligned}
$$




$$
\begin{aligned}
& =e^{-\lambda t}\left[\mathcal{C}(t) x-\frac{t^{n}}{n !} x\right]+\lambda e^{-\lambda t} \int_{0}^{t} \mathcal{C}(s) x d s \\
& +\lambda^{2} \int_{0}^{t} e^{-\lambda s}\left[\int_{0}^{s} \mathcal{C}(s) x d r\right] d s-\lambda \int_{0}^{t} e^{-\lambda s} \frac{s^{n}}{n !} x d s \\
& =\lambda^{2} R_{\lambda}(t) x+\lambda e^{-\lambda t} \int_{0}^{t} \mathcal{C}(s) x d s \\
& +e^{-\lambda t} \mathcal{C}(t) x-\int_{0}^{t} e^{-\lambda s} \frac{s^{n-1}}{(n-1) !} x d s .
\end{aligned}
$$

Set $\mathcal{C}_{\lambda}(t) x:=\int_{0}^{t} \mathcal{C}(s) x d s+\lambda^{-1} \mathcal{C}(t) x$. Then (1.6) implies that

$$
\left(\lambda^{2}-A\right) R_{\lambda}(t) x=\lambda e^{-\lambda t}\left[g_{\lambda}(t) x-\mathcal{C}_{\lambda}(t) x\right]
$$

where

$$
\begin{aligned}
g_{\lambda}(t) & :=\frac{1}{\lambda} \int_{0}^{t} e^{\lambda(t-s)} \frac{s^{n-1}}{(n-1) !} d s \\
& =\frac{e^{\lambda t}}{\lambda^{n+1}}-\frac{1}{\lambda^{n+1}}-\frac{t}{\lambda^{n}}-\frac{t^{2}}{2 ! \lambda^{n-1}}-\cdots-\frac{t^{n-1}}{(n-1) ! \lambda^{2}} .
\end{aligned}
$$

From $(1.7),(1.8)$ and the definition of $\mathcal{C}_{\lambda}(t) x$, together with an argument similar to that of $\left[2\right.$, Proposition 2.5], we can prove that there exists $\omega_{0}>0$ such that $\left(\omega_{0}^{2}, \infty\right) \subset \varrho(A)$. This implies that $\lambda^{2}-A$ has bounded inverse for $\lambda>\omega_{0}$.

A more precise result than Lemma 1.8 was stated in [13] without proof. In the following we write $\mathcal{R}(\lambda, A):=\left(\lambda^{2}-A\right)^{-1}$ for $\lambda>\omega_{0}$.

Proof of Theorem 1.7. [15, Theorem 1.5 and Proposition 1.8] implies that (i), (ii) and (iii) of Theorem 1.7 are equivalent for a not necessarily exponentially bounded global $n$-times regularized cosine operator family with $A$ as a subgenerator (see [15, Definition 1.3]); it is easy to see that the proof there is valid for our local case. Therefore it remains to show that $A$ is the generator of $\{\mathcal{C}(t)\}_{t \in[0, \tau)}$ under the equivalent conditions (i)-(iii). To do this it suffices to prove that if $x, z \in X$ satisfy

$$
\int_{0}^{t}(t-s) \mathcal{C}(s) z d s=\mathcal{C}(t) x-\frac{t^{n}}{n !} x,
$$

then $x \in D(A)$ and $A x=z$. Now (ii)(a) implies $\mathcal{C}(t) \mathcal{R}(\lambda, A)=\mathcal{R}(\lambda, A) \mathcal{C}(t)$ for all $\lambda^{2} \in \varrho(A)$. Hence 


$$
\begin{aligned}
& \int_{0}^{t}(t-s) \mathcal{C}(s) \mathcal{R}(\lambda, A) z d s=\mathcal{R}(\lambda, A) \int_{0}^{t}(t-s) \mathcal{C}(s) z d s \\
& =\mathcal{R}(\lambda, A) \mathcal{C}(t) x-\frac{t^{n}}{n !} \mathcal{R}(\lambda, A) x=\mathcal{C}(t) \mathcal{R}(\lambda, A) x-\frac{t^{n}}{n !} \mathcal{R}(\lambda, A) x \\
& =A \int_{0}^{t}(t-s) \mathcal{C}(s) \mathcal{R}(\lambda, A) x d s=\int_{0}^{t}(t-s) \mathcal{C}(s) A \mathcal{R}(\lambda, A) x d s
\end{aligned}
$$

Differentiate both sides with respect to $t$ twice to find $\mathcal{C}(t) \mathcal{R}(\lambda, A) z=$ $\mathcal{C}(t) A \mathcal{R}(\lambda, A) x$. Since $\{\mathcal{C}(t)\}_{t \in[0, \tau)}$ is nondegenerate, it follows that $\mathcal{R}(\lambda, A) z$ $=A \mathcal{R}(\lambda, A) x$. This gives $A \mathcal{R}(\lambda, A) x \in D(A)$ and hence $\mathcal{R}(\lambda, A) x \in D\left(A^{2}\right)$. Consequently, $x=\left(\lambda^{2}-A\right) \mathcal{R}(\lambda, A) x \in D(A)$ and $A x=z$.

When the equivalent conditions (i)-(iii) of Theorem 1.7 hold, we may apply the method used in [11, Theorems 4.2 and 7.6] or [17, Theorem 5.2] to show that (iv) is true. Here we mention that (iv) is not necessary in what follows. We include it only for completeness.

2. Bounded perturbations. In this section, we study bounded perturbations for $n$-times integrated semigroups. Let $f(\cdot)$ be a given function and define

$$
\begin{aligned}
J^{k}(f)(t) & =\int_{0}^{t} \int_{0}^{t_{1}} \cdots \int_{0}^{t_{k-1}} f\left(t_{k}\right) d t_{k} d t_{k-1} \cdots d t_{1} \\
& =\frac{1}{(k-1) !} \int_{0}^{t}\left(t-t_{k}\right)^{k-1} f\left(t_{k}\right) d t_{k}
\end{aligned}
$$

Lemma 2.1. For $B \in L(X)$ and $n \in \mathbb{N}$,

$$
\sum_{k=0}^{n} C_{n}^{k}(-B)^{k} J^{k+1}\left[e^{t B} \frac{t^{n-1}}{(n-1) !}\right] x=\frac{t^{n}}{n !} x \quad \forall x \in X
$$

where $C_{n}^{k}=\frac{n !}{k !(n-k) !}$.

Proof. We first show the following relation for $0 \leq k \leq n-1$ :

$$
\begin{aligned}
B^{k} J^{k+1}\left[e^{t B} \frac{t^{n-1}}{(n-1) !}\right] x & \\
& =\sum_{j=0}^{k}(-1)^{j} C_{k}^{j} J^{j+1}\left[e^{t B} \frac{t^{n-j-1}}{(n-j-1) !}\right] x \quad \forall x \in X .
\end{aligned}
$$

(2.1) is true for $k=0$. Assume that it is true for $0<k<n-1$. Applying integration by parts and making use of $C_{k}^{j}+C_{k}^{j-1}=C_{k+1}^{j}$ and the convention $C_{k}^{-1}=0$ we find that 


$$
\begin{aligned}
B^{k+1} & J^{k+2}\left[e^{t B} \frac{t^{n-1}}{(n-1) !}\right] x=B J\left\{B^{k} J^{k+1}\left[e^{t B} \frac{t^{n-1}}{(n-1) !}\right] x\right\} \\
= & B J\left\{\sum_{j=0}^{k}(-1)^{j} C_{k}^{j} J^{j+1}\left[e^{t B} \frac{t^{n-j-1}}{(n-j-1) !}\right] x\right\} \\
= & \sum_{j=0}^{k}(-1)^{j} C_{k}^{j} J^{j+1} B J\left[e^{t B} \frac{t^{n-j-1}}{(n-j-1) !}\right] x \\
= & \sum_{j=0}^{k}(-1)^{j} C_{k}^{j} J^{j+1}\left[e^{t B} \frac{t^{n-j-1}}{(n-j-1) !}-J\left(e^{t B} \frac{t^{n-j-2}}{(n-j-2) !}\right)\right] x \\
= & \sum_{j=0}^{k}(-1)^{j}\left[C_{k}^{j}+C_{k}^{j-1}\right] J^{j+1}\left[e^{t B} \frac{t^{n-j-1}}{(n-j-1) !}\right] x \\
& +(-1)^{k+1} J^{k+2}\left[e^{t B} \frac{\left.t^{n-k-2}\right]}{(n-k-2) !}\right] x \\
= & \sum_{j=0}^{k+1}(-1)^{j} C_{k+1}^{j} J^{j+1}\left[e^{t B} \frac{t^{n-j-1}}{(n-j-1) !}\right] x,
\end{aligned}
$$

and (2.1) follows. Next we show that

$$
\begin{aligned}
B^{n} J^{n+1}\left[e^{t B}\right. & \left.\frac{t^{n-1}}{(n-1) !}\right] x \\
& =\sum_{j=0}^{n-1}(-1)^{j} C_{n}^{j} J^{j+1}\left[e^{t B} \frac{t^{n-j-1}}{(n-j-1) !}\right] x+(-1)^{n} \frac{t^{n}}{n !} x .
\end{aligned}
$$

Consider the left side of (2.1) for $k=n-1$. Make use of (2.2) and apply integration by parts to find

$$
\begin{aligned}
B^{n} J^{n+1} & {\left[e^{t B} \frac{t^{n-1}}{(n-1) !}\right] x=B J\left\{B^{n-1} J^{n}\left[e^{t B} \frac{t^{n-1}}{(n-1) !}\right] x\right\} } \\
= & B J \sum_{j=0}^{n-1}(-1)^{j} C_{n-1}^{j} J^{j+1}\left[e^{t B} \frac{t^{n-j-1}}{(n-j-1) !}\right] x \\
= & (-1)^{n-1} B\left[J^{n+1} e^{t B}\right] x \\
& +\sum_{j=0}^{n-2}(-1)^{j} C_{n-1}^{j} J^{j+1}\left[e^{t B} \frac{t^{n-j-1}}{(n-j-1) !}-J e^{t B} \frac{t^{n-j-2}}{(n-j-2) !}\right] x
\end{aligned}
$$




$$
\begin{aligned}
& =\sum_{j=0}^{n-1}(-1)^{j}\left[C_{n-1}^{j}+C_{n-1}^{j-1}\right] J^{j+1} e^{t B} \frac{t^{n-j-1}}{(n-j-1) !} x+(-1)^{n} J^{n} x \\
& =\sum_{j=0}^{n-1}(-1)^{j} C_{n}^{j} J^{j+1}\left[e^{t B} \frac{t^{n-j-1}}{(n-j-1) !}\right] x+(-1)^{n} \frac{t^{n}}{n !} x,
\end{aligned}
$$

so (2.3) is true. Here we make use of $C_{n-1}^{j}+C_{n-1}^{j-1}=C_{n}^{j}$ and the convention $C_{n-1}^{-1}=0$.

From (2.1), (2.3) and the relations

$$
\sum_{k=0}^{n-j}(-1)^{k} C_{n-j}^{k}=0 \quad \forall 0 \leq j \leq n-1, \quad C_{n}^{k} C_{k}^{j}=C_{n}^{j} C_{n-j}^{k}
$$

we have, for all $x \in X$,

$$
\begin{aligned}
\sum_{k=0}^{n} C_{n}^{k}(-B)^{k} J^{k+1} e^{t B} \frac{t^{n-1}}{(n-1) !} x & \sum_{k=0}^{n-1} C_{n}^{k}(-1)^{k} \sum_{j=0}^{k}(-1)^{j} C_{k}^{j} J^{j+1} e^{t B} \frac{t^{n-j-1}}{(n-j-1) !} x \\
& +\sum_{j=0}^{n-1}(-1)^{n+j} C_{n}^{j} J^{j+1} e^{t B} \frac{t^{n-j-1}}{(n-j-1) !} x+\frac{t^{n}}{n !} x \\
= & \sum_{j=0}^{n-1}(-1)^{j}\left\{\left[\sum_{k=j}^{n-1}(-1)^{k} C_{n}^{k} C_{k}^{j}+(-1)^{n} C_{n}^{j}\right] J^{j+1}\left[e^{t B} \frac{t^{n-j-1}}{(n-j-1) !}\right] x\right\}+\frac{t^{n}}{n !} x \\
= & \sum_{j=0}^{n-1} C_{n}^{j}\left\{\left[\sum_{k=0}^{n-j}(-1)^{k} C_{n-j}^{k}\right] J^{j+1}\left[e^{t B} \frac{t^{n-j-1}}{(n-j-1) !}\right] x\right\}+\frac{t^{n}}{n !} x=\frac{t^{n}}{n !} x .
\end{aligned}
$$

The following lemma is an immediate consequence of (ii) of Theorem 1.3 and integration by parts.

Lemma 2.2. Assume $B \in L(X)$ and $A$ is the generator of $\{S(t)\}_{t \in[0, \tau)}$. If $B A \subseteq A B$ then $\int_{0}^{t} e^{s B} S(s) x d s \in D(A)$ and

$$
\begin{aligned}
A \int_{0}^{t} e^{s B} S(s) x d s= & e^{t B}\left[S(t) x-\frac{t^{n}}{n !} x\right] \\
& -B \int_{0}^{t} e^{s B}\left[S(s)-\frac{s^{n}}{n !}\right] x d s \quad \forall x \in X, t \in[0, \tau) .
\end{aligned}
$$


TheOrem 2.3. Assume that $\{S(t)\}_{t \in[0, \tau)}$ is an n-times integrated semigroup generated by $A$ and that $B \in L(X)$ with $B A \subseteq A B$. Then $A+B$ generates an $n$-times integrated semigroup given by

$$
\begin{aligned}
S_{B}(t) x & :=e^{B t} S(t) x+\int_{0}^{t} P(t-s) e^{s B} S(s) x d s, \\
& =\sum_{k=0}^{n} C_{n}^{k}(-B)^{k} J^{k} e^{t B} S(t) x \quad \forall x \in X, t \in[0, \tau),
\end{aligned}
$$

where $P(t)=\sum_{k=0}^{n} \frac{(-1)^{k} t^{k-1}}{(k-1) !} C_{n}^{k} B^{k}$ (see also $[2,16]$ for the exponentially bounded case).

Proof. Let $x \in X$. From Lemma 2.2,

$$
\begin{aligned}
A \int_{0}^{t} S_{B}(s) x d s= & \sum_{k=0}^{n} C_{n}^{k}(-B)^{k} A J^{k+1} e^{t B} S(t) x \\
= & \sum_{k=0}^{n} C_{n}^{k}(-B)^{k} J^{k} A \int_{0}^{t} e^{s B} S(s) x d s \\
= & \sum_{k=0}^{n} C_{n}^{k}(-B)^{k} J^{k} e^{t B}\left[S(t)-\frac{t^{n}}{n !}\right] x \\
& +\sum_{k=0}^{n} C_{n}^{k}(-B)^{k+1} J^{k+1} e^{t B}\left[S(t)-\frac{t^{n}}{n !}\right] x \\
= & S_{B}(t) x-B \int_{0}^{t} S_{B}(s) x d s-\Psi_{1}(t) x-\Psi_{2}(t) x,
\end{aligned}
$$

where

$$
\begin{aligned}
& \Psi_{1}(t):=\sum_{k=0}^{n} C_{n}^{k}(-B)^{k} J^{k}\left[e^{t B} \frac{t^{n}}{n !}\right], \\
& \Psi_{2}(t):=\sum_{k=0}^{n} C_{n}^{k}(-B)^{k+1} J^{k+1}\left[e^{t B} \frac{t^{n}}{n !}\right] .
\end{aligned}
$$

(2.4) gives us

$$
(A+B) \int_{0}^{t} S_{B}(s) x d s=S_{B}(t) x-\Psi_{1}(t) x-\Psi_{2}(t) x .
$$

Apply integration by parts to $\Psi_{2}(t)$ and then use Lemma 2.1 to find

$$
\Psi_{2}(t) x=-\Psi_{1}(t) x+\sum_{k=0}^{n} C_{n}^{k}(-B)^{k} J^{k+1}\left[e^{t B} \frac{t^{n-1}}{(n-1) !}\right] x=-\Psi_{1}(t) x+\frac{t^{n}}{n !} x .
$$


This, together with (2.5), gives

$$
(A+B) \int_{0}^{t} S_{B}(s) x d s=S_{B}(t) x-\frac{t^{n}}{n !} x \quad \forall x \in X, t \in[0, \tau) .
$$

It is easy to see from $(2.6)$ that $\left\{S_{B}(t)\right\}_{t \in[0, \tau)}$ is nondegenerate. Its expression and the relation $B A \subseteq A B$, together with Proposition 1.4(iii), imply that $S_{B}(t)(A+B) \subseteq(A+B) S_{B}(t)$. By Theorem 1.3, $\left\{S_{B}(t)\right\}_{t \in[0, \tau)}$ is an $n$-times integrated semigroup generated by $A+B$.

By the expression of $\left\{S_{B}(t)\right\}_{t \in[0, \tau)}$ in Theorem 2.3, if $\tau=\infty$ and $\{S(t)\}_{t \in[0, \infty)}$ is exponentially bounded then so is $\left\{S_{B}(t)\right\}_{t \in[0, \infty)}$.

Before proving the following theorem, we first note that if $B \in L(X)$ satisfies $\operatorname{Im}(B) \subseteq D\left(A^{n}\right)$ then the equality

$$
S^{(n)}(t) B x=S(t) A^{n} B x+\sum_{k=0}^{n-1} \frac{t^{k}}{k !} A^{k} B x \quad \forall x \in X, t \in[0, \tau)
$$

is an easy consequence of Proposition 1.4 and hence $S^{(n)}(t) B$ is bounded for every $t \in[0, \tau)$ and strongly continuous on $[0, \tau)$.

TheOREM 2.4. Let $\{S(t)\}_{t \in[0, \tau)}$ be an n-times integrated semigroup generated by $A$ and let $B \in L(X)$ be such that $\operatorname{Im}(B) \subseteq D\left(A^{n}\right)$. Then, for all $x \in X$, the following equation has a unique strongly continuous solution:

$$
V(t) x=S(t) x+\int_{0}^{t} S^{(n)}(t-s) B V(s) x d s \quad \forall t \in[0, \tau) .
$$

Proof. Set $V_{0}(t)=S(t)$ and assume inductively that $V_{m-1}(t) \in L(X)$ is well defined for $t \in[0, \tau)$ and that $V_{m-1}(\cdot) x \in C([0, \tau), X)$ for every $x \in X$. We now define $V_{m}(t)$ by

$$
V_{m}(t) x=\int_{0}^{t} S^{(n)}(t-s) B V_{m-1}(s) x d s .
$$

Then it is easy to verify that $V_{m}(t) \in L(X)$ for every $t \in[0, \tau)$ and $V_{m}(\cdot) x \in$ $C([0, \tau), X)$ for every $x \in X$.

For given $t \in[0, \tau)$ define

$$
M(t):=\sup _{0 \leq s \leq t}\left\{\left\|S^{(n)}(s) B\right\|,\|S(s)\|\right\} .
$$

Then $M(t) \geq 0$ is finite for every $t \in[0, \tau)$ and increasing on $[0, \tau)$. We now claim that

$$
\left\|V_{m}(t)\right\| \leq \frac{M(t)^{m+1} t^{m}}{m !} \quad \forall m \in \mathbb{N} \cup\{0\}, t \in[0, \tau) .
$$


Indeed, (2.9) is true for $m=0$. Assume it is true for $m$ replaced by $m-1$. From (2.8) we have

$$
\left\|V_{m}(t)\right\| \leq \int_{0}^{t} \frac{M(t-s) M^{m}(s) s^{m-1}}{m !} d s \leq \frac{M(t)^{m+1} t^{m}}{m !} .
$$

Hence (2.9) is true for all $m \in \mathbb{N} \cup\{0\}$ and $t \in[0, \tau)$. Define

$$
S_{B}(t):=\sum_{m=0}^{\infty} V_{m}(t)
$$

Then (2.9) implies that the series converges uniformly on every closed subinterval $[0, b] \subseteq[0, \tau)$ in the uniform operator topology. Since

$$
\begin{aligned}
S_{B}(t) x & =S(t) x+\int_{0}^{t} S^{(n)}(t-s) B \sum_{m=0}^{\infty} V_{m}(s) x d s \\
& =S(t) x+\int_{0}^{t} S^{(n)}(t-s) B S_{B}(s) x d s,
\end{aligned}
$$

$S_{B}(\cdot)$ satisfies $(2.7)$ and is strongly continuous on $[0, \tau)$.

To prove the uniqueness, it suffices to show that a continuous $X$-valued function $v(\cdot)$ vanishes if it satisfies

$$
v(t)=\int_{0}^{t} S^{(n)}(t-s) B v(s) d s \quad \forall t \in[0, \tau) .
$$

For given $t \in[0, \tau)$, set $N(t):=\sup _{0 \leq s \leq t}\|v(s)\|$. Then

$$
\|v(t)\| \leq \int_{0}^{t} M(t-s)\|v(s)\| d s \leq N(t) M(t) t .
$$

Repeating the above process, we show that

$$
\|v(t)\| \leq N(t) \frac{M(t)^{m} t^{m}}{m !} \quad \forall t \in[0, \tau), m \in \mathbb{N} .
$$

Let $m \rightarrow \infty$ to find $v(t) \equiv 0$ for all $t \in[0, \tau)$.

THEOREM 2.5. The strongly continuous family of operators $\left\{S_{B}(t)\right\}_{t \in[0, \tau)}$, defined in Theorem 2.4, is an n-times integrated semigroup generated by $A+B$.

Proof. From Proposition 1.4(i),

$$
\begin{aligned}
A \int_{0}^{t}\left[\int_{0}^{s} S^{(n)}(s-r) B S_{B}(r) x d r\right] d s & =\int_{0}^{t} A\left[\int_{r}^{t} S^{(n)}(s-r) B S_{B}(r) x d s\right] d r \\
& =\int_{0}^{t} A\left[S^{(n-1)}(t-r) B S_{B}(r) x\right] d r
\end{aligned}
$$




$$
\begin{aligned}
& =\int_{0}^{t}\left[S^{(n)}(t-r) B S_{B}(r) x-B S_{B}(r) x\right] d r \\
& =\int_{0}^{t} S^{(n)}(t-r) B S_{B}(r) x d r-B \int_{0}^{t} S_{B}(r) x d r .
\end{aligned}
$$

This implies that

$$
\begin{aligned}
& (A+B) \int_{0}^{t} S_{B}(s) x d s \\
& \quad=A \int_{0}^{t} S(s) x d s+A \int_{0}^{t}\left[\int_{0}^{s} S^{(n)}(s-r) B S_{B}(r) x d r\right] d s \\
& \quad=S(t) x-\frac{t^{n}}{n !} x+\int_{0}^{t} S^{(n)}(t-r) B S_{B}(r) x d r=S_{B}(t) x-\frac{t^{n}}{n !} x
\end{aligned}
$$

and hence $\left\{S_{B}(t)\right\}_{t \in[0, \tau)}$ is nondegenerate.

In the following we show that the solutions of the following local first order abstract differential equation are unique:

$$
u^{\prime}(t)=(A+B) u(t), \quad t \in[0, \tau) ; \quad u(0)=x .
$$

Let $u(\cdot)$ be a solution of $(2.10)$ with $u(0)=0$. Define

$$
v(t):=\frac{1}{n !} \int_{0}^{t}(t-s)^{n} u(s) d s \quad \forall t \in[0, \tau) .
$$

Then $v(\cdot)$ is $(n+1)$-times continuously differentiable and satisfies (2.10) with $v(0)=0$. If we can show that $v(t) \equiv 0$ then the same is true for $u(t)$ and hence the solutions of (2.10) are unique. It is easily seen that $v(t) \in D\left(A^{n+1}\right)$ for all $t \in[0, \tau)$. Thus we have, by Proposition 1.4(i),

$$
\begin{aligned}
\int_{0}^{t} S^{(n)}(t-s) B v(s) d s=\int_{0}^{t} S^{(n)}(t-s) v^{\prime}(s) d s-\int_{0}^{t} S^{(n)}(t-s) A v(s) d s \\
=\int_{0}^{t} S^{(n)}(t-s) d v(s)-\int_{0}^{t} A S^{(n)}(t-s) v(s) d s \\
=v(t)+\int_{0}^{t} S^{(n+1)}(t-s) v(s) d s-\int_{0}^{t} S^{(n+1)}(t-s) v(s) d s=v(t) .
\end{aligned}
$$

From the last part of the proof of Theorem 2.4, $v(t) \equiv 0$ for all $t \in[0, \tau)$. Therefore the solutions of (2.10) are unique. An application of Theorem 1.3 shows that $\left\{S_{B}(t)\right\}_{t \in[0, \tau)}$ is the $n$-times integrated semigroup generated by $A+B$. 
COROllary 2.6. Under the conditions of Theorem 2.4, if the n-times integrated semigroup $\{S(t)\}_{t \in[0, \infty)}$ is exponentially bounded then so is $\left\{S_{B}(t)\right\}_{t \in[0, \infty)}$.

Proof. From Theorem 2.4 and [16, Theorem 2.3], the following is true:

$$
\breve{S}_{B}(t):=S(t)+\sum_{m=1}^{\infty}\left[S^{(n)}(t) B\right]^{m} S(t)=\sum_{m=0}^{\infty} V_{m}(t)=S_{B}(t) .
$$

From [16, Theorem 2.3] again, $\left\{S_{B}(t)\right\}_{t \in[0, \infty)}$ is exponentially bounded.

It follows from Theorems 2.3, 2.5 and Corollary 2.6 that if $A+B$ generates an (exponentially bounded) $n$-times integrated semigroup then so does $A$.

3. Unbounded perturbations. This section is devoted to the study of unbounded perturbations for $n$-times integrated semigroups. Two cases will be considered.

TheOrem 3.1. Assume $\{S(t)\}_{t \in[0, \tau)}$ is an n-times integrated semigroup generated by $A$, and let $B \in L([D(A)])$ with $\operatorname{Im}(B) \subseteq D\left(A^{n+1}\right)$. If $A B x=$ $B A x$ for all $x \in D\left(A^{2}\right)$, then $A+B$ generates an $n$-times integrated semigroup $\left\{S_{B}(t)\right\}_{t \in[0, \tau)}$ on $X$ that is the unique solution of the equation

$$
\begin{aligned}
& S_{B}(t) x=S(t) x \\
& \quad+\int_{0}^{t} S^{(n)}(t-s)\left(\lambda_{0}-A\right) B R\left(\lambda_{0}, A\right) S_{B}(s) x d s \quad \forall x \in X, t \in[0, \tau),
\end{aligned}
$$

for any $\lambda_{0} \in \varrho(A)$.

Proof. We first show that $A+B$ is closed on $X$. To do this, it suffices to show that $\lambda_{0}-(A+B)$ is closed. From the relation $A B x=B A x\left(x \in D\left(A^{2}\right)\right)$, we have $R\left(\lambda_{0}, A\right) B x=B R\left(\lambda_{0}, A\right) x$ for all $x \in D(A)$. Let $x_{m} \in D(A)$ and assume that $x_{m} \rightarrow x$ and $y_{m}=\left[\lambda_{0}-(A+B)\right] x_{m} \rightarrow y$ in $X$. Then the following limit holds in $[D(A)]$ :

$$
\begin{aligned}
x_{m} & =R\left(\lambda_{0}, A\right) y_{m}+R\left(\lambda_{0}, A\right) B x_{m} \\
& =R\left(\lambda_{0}, A\right) y_{m}+B R\left(\lambda_{0}, A\right) x_{m} \rightarrow R\left(\lambda_{0}, A\right) y+B R\left(\lambda_{0}, A\right) x .
\end{aligned}
$$

Therefore $x \in D(A)$ and $R\left(\lambda_{0}, A\right) y+B R\left(\lambda_{0}, A\right) x=x$, or equivalently, $\left[\lambda_{0}-(A+B)\right] x=y$, as desired. For $x \in X$ and $t \in[0, \tau)$, Proposition 1.4(i) gives

$$
\begin{aligned}
& S^{(n)}(t)\left(\lambda_{0}-A\right) B R\left(\lambda_{0}, A\right) x \\
& \quad=S(t) A^{n}\left(\lambda_{0}-A\right) B R\left(\lambda_{0}, A\right) x+\sum_{k=0}^{n-1} \frac{t^{k}}{k !} A^{k}\left(\lambda_{0}-A\right) B R\left(\lambda_{0}, A\right) x .
\end{aligned}
$$


Since $D\left(A^{k}\left(\lambda_{0}-A\right) B R\left(\lambda_{0}, A\right)\right)=X$ by induction on $k$, we may show that $A^{k}\left(\lambda_{0}-A\right) B R\left(\lambda_{0}, A\right)$ is closed for $0 \leq k \leq n$ and hence bounded. This and the foregoing imply that

$$
S^{(n)}(t)\left(\lambda_{0}-A\right) B R\left(\lambda_{0}, A\right) \in L(X) \quad \forall t \in[0, \tau)
$$

and that

$$
S^{(n)}(\cdot)\left(\lambda_{0}-A\right) B R\left(\lambda_{0}, A\right) x \in C([0, \tau), X) \quad \forall x \in X .
$$

Define

$$
\begin{gathered}
V_{0}(t) x:=S(t) x \quad \forall x \in X, t \in[0, \tau), \\
V_{m}(t) x:=\int_{0}^{t} S^{(n)}(t-s)\left(\lambda_{0}-A\right) B R\left(\lambda_{0}, A\right) V_{m-1}(s) x d s \quad \forall x \in X, t \in[0, \tau),
\end{gathered}
$$

where $m \in \mathbb{N}$. From (3.2) and (3.3), we have $V_{m}(\cdot) x \in C([0, \tau), X)$ for all $x \in X$ and $m \in \mathbb{N} \cup\{0\}$. For $t \in[0, \tau)$, define

$$
M(t)=\sup _{0 \leq s \leq t}\left\{\|S(s)\|,\left\|S^{(n)}(s)\left(\lambda_{0}-A\right) B R\left(\lambda_{0}, A\right)\right\|\right\} .
$$

Then we have the following inequality, similar to $(2.9)$ :

$$
\left\|V_{m}(t)\right\| \leq \frac{M^{m+1}(t) t^{m}}{m !} \quad \forall m \in \mathbb{N} \cup\{0\} .
$$

Define

$$
S_{B}(t):=\sum_{m=0}^{\infty} V_{m}(t) .
$$

(3.4) implies that the series converges uniformly on every closed subinterval $[0, b] \subset[0, \tau)$ in the uniform operator topology. Since

$$
\begin{aligned}
S_{B}(t) & =\sum_{m=0}^{\infty} V_{m}(t) \\
& =S(t)+\int_{0}^{t} S^{(n)}(t-s)\left(\lambda_{0}-A\right) B R\left(\lambda_{0}, A\right) \sum_{m=0}^{\infty} V_{m}(s) d s \\
& =S(t)+\int_{0}^{t} S^{(n)}(t-s)\left(\lambda_{0}-A\right) B R\left(\lambda_{0}, A\right) S_{B}(s) d s,
\end{aligned}
$$

$S_{B}(\cdot)$ is a solution of (3.1). From (3.5) and Proposition 1.4(i),

$$
\begin{aligned}
A \int_{0}^{t} S_{B}(s) x d s= & A \int_{0}^{t} S(s) x d s \\
& +A \int_{0}^{t}\left[\int_{0}^{s} S^{(n)}(s-r)\left(\lambda_{0}-A\right) B R\left(\lambda_{0}, A\right) S_{B}(r) x d r\right] d s
\end{aligned}
$$




$$
\begin{aligned}
& =S(t) x-\frac{t^{n}}{n !} x+A \int_{0}^{t} S^{(n-1)}(t-r)\left(\lambda_{0}-A\right) B R\left(\lambda_{0}, A\right) S_{B}(r) x d r \\
& =S_{B}(t) x+\int_{0}^{t} S^{(n)}(t-r)\left(\lambda_{0}-A\right) B R\left(\lambda_{0}, A\right) S_{B}(r) x d r \\
& \quad-\int_{0}^{t}\left(\lambda_{0}-A\right) B R\left(\lambda_{0}, A\right) S_{B}(r) x d r-\frac{t^{n}}{n !} x .
\end{aligned}
$$

The relations $\int_{0}^{t} S_{B}(s) x d s \in D(A)$ for every $x \in X$ and $\left(\lambda_{0}-A\right) B R\left(\lambda_{0}, A\right) x$ $=B x$ for every $x \in D(A)$ imply that

$$
\begin{aligned}
\int_{0}^{t}\left(\lambda_{0}-A\right) B R\left(\lambda_{0}, A\right) & S_{B}(s) x d s \\
& =\left(\lambda_{0}-A\right) B R\left(\lambda_{0}, A\right) \int_{0}^{t} S_{B}(s) x d s=B \int_{0}^{t} S_{B}(s) x d s .
\end{aligned}
$$

This, together with (3.6), gives

$$
(A+B) \int_{0}^{t} S_{B}(s) x d s=S_{B}(t) x-\frac{t^{n}}{n !} x \quad \forall x \in X .
$$

The uniqueness of solutions of (3.1) may be proved in the same way employed in Theorem 2.4. As regards the uniqueness of solutions of (2.10) with $A$ and $B$ given in this theorem, we may prove it in the same way employed in Theorem 2.5. Thus an application of Theorem 1.3 shows that $\left\{S_{B}(t)\right\}_{t \in[0, \tau)}$ is the $n$-times integrated semigroup generated by $A+B$.

Instead of the relation $A B x=B A x$ for all $x \in D\left(A^{2}\right)$ in Theorem 3.1, in the following theorem, we assume directly that $A+B$ is closed. So far we do not know if it is automatically true.

ThEOREM 3.2. Assume $\{S(t)\}_{t \in[0, \tau)}$ is an n-times integrated semigroup generated by $A$, and $B \in L([D(A)])$ with $\operatorname{Im}(B) \subseteq D\left(A^{n+1}\right)$. If $A+B$ is closed on $X$ then $A+B$ generates an $n$-times integrated semigroup $\left\{S_{B}(t)\right\}_{t \in[0, \tau)}$ on $X$ that is the unique solution of the equation

$$
\begin{aligned}
S_{B}(t) x= & S(t) x+B \int_{0}^{t} S_{B}(s) x d s \\
& +\int_{0}^{t} S^{(n+1)}(t-s) B\left[\int_{0}^{s} S_{B}(r) x d r\right] d s \quad \forall x \in X, t \in[0, \tau) .
\end{aligned}
$$


Proof. First of all, we may show, by induction on $k$, that $A^{k} B \in L([D(A)])$ for $1 \leq k \leq n$. On the other hand, it is routine to show that $\left.S(t)\right|_{D(A)}$ is in $L([D(A)])$ for every $t \in[0, \tau)$ and that $\left\{\left.S(t)\right|_{D(A)}\right\}_{t \in[0, \tau)}$ is strongly continuous in $[D(A)]$. Thus the relation

$$
S^{(n)}(t) B x=S(t) A^{n} B x+\sum_{k=0}^{n-1} \frac{t^{k}}{k !} A^{k} B x \quad \forall x \in D(A), t \in[0, \tau)
$$

implies that $S^{(n)}(\cdot) B x \in C([0, \tau),[D(A)])$ for every $x \in D(A)$. Moreover, by differentiating both sides of $(3.9)$, we have $S^{(n+1)}(t) B x \in L([D(A)], X)$ for every $t \in[0, \tau)$ and $S^{(n+1)}(\cdot) B x \in C([0, \tau), X)$ for every $x \in D(A)$.

Define

$$
\begin{aligned}
V_{0}(t) x & :=\int_{0}^{t} S(s) x d s \quad \forall x \in X, t \in[0, \tau), \\
V_{m}(t) x & :=\int_{0}^{t} S^{(n)}(t-s) B V_{m-1}(s) x d s \quad \forall x \in X, t \in[0, \tau) .
\end{aligned}
$$

We show that for every $x \in X$ and $m \in \mathbb{N}, V_{m}(\cdot) x \in C([0, \tau),[D(A)])$. The assertion is true for $m=0$. Now assume it is true for $m-1$. Then $B V_{m-1}(\cdot) x$ makes sense for every $x \in X$, and $B V_{m-1}(\cdot) x \in C([0, \tau),[D(A)])$. From Proposition 1.4(i), $A S^{(n)}(t-s) B V_{m-1}(s) x=S^{(n+1)}(t-s) B V_{m-1}(s) x$. This, combined with the properties of $S^{(n+1)}(\cdot) B x$, implies that $V_{m}(\cdot) x \in$ $C([0, \tau),[D(A)])$ by its definition, and hence the assertion is true for $m \in \mathbb{N}$.

For $t \in[0, \tau)$, define

$$
\begin{aligned}
& M_{1}(t):=\sup _{0 \leq s \leq t}\left\{\sup \left\{\left\|\int_{0}^{s} S(r) x d r\right\|_{[D(A)]}: x \in X,\|x\| \leq 1\right\}\right\}, \\
& M_{2}(t):=\sup _{0 \leq s \leq t}\left\{\left\|S^{(n)}(s) B\right\|_{L([D(A)])}\right\},
\end{aligned}
$$

and

$$
M(t):=\max \left\{M_{1}(t), M_{2}(t)\right\} .
$$

Then we can show that

$$
\left\|V_{m}(t)\right\|_{L(X,[D(A)])} \leq \frac{M^{k+1}(t) t^{k}}{k !}
$$

and hence the series $\widetilde{S}_{B}(t):=\sum_{m=0}^{\infty} V_{m}(t)$ converges uniformly on every closed subinterval $[0, b] \subset[0, \tau)$ in $L(X,[D(A)])$. Clearly, $\widetilde{S}_{B}(\cdot)$ satisfies

$$
\widetilde{S}_{B}(t) x=\int_{0}^{t} S(s) x d s+\int_{0}^{t} S^{(n)}(t-s) B \widetilde{S}_{B}(s) x d s \quad \forall x \in X, t \in[0, \tau),
$$


where the second integral converges in the norm topology of $[D(A)]$. Moreover, from

$$
\begin{aligned}
A \int_{0}^{t} \widetilde{S}_{B}(s) x d s= & A \int_{0}^{t} \int_{0}^{s} S(r) x d r d s+A \int_{0}^{t}\left[\int_{0}^{s} S^{(n)}(s-r) B \widetilde{S}_{B}(r) x d r\right] d s \\
= & \int_{0}^{t} S(s) x d s-\frac{t^{n+1}}{(n+1) !} x+A \int_{0}^{t} S^{(n-1)}(t-r) B \widetilde{S}_{B}(r) x d r \\
= & \int_{0}^{t} S(s) x d s+\int_{0}^{t} S^{(n)}(t-r) B \widetilde{S}_{B}(r) x d r \\
& -\int_{0}^{t} B \widetilde{S}_{B}(r) x d r-\frac{t^{n+1}}{(n+1) !} x
\end{aligned}
$$

the following relation holds:

$$
(A+B) \int_{0}^{t} \widetilde{S}_{B}(s) x d s=\widetilde{S}_{B}(t) x-\frac{t^{n+1}}{(n+1) !} x \quad \forall x \in X, t \in[0, \tau) .
$$

Using the uniqueness argument employed in Theorems 2.4 and 2.5 we may also show that the solutions of (2.10) are unique for the operators $A$ and $B$ given in this theorem and hence $\left\{\widetilde{S}_{B}(t)\right\}_{t \in[0, \tau)}$ is a nondegenerate $(n+1)$ times integrated semigroup generated by $A+B$ by Theorem 1.3.

From (3.10), for every $x \in X, \widetilde{S}_{B}(t) x$ is continuously differentiable with respect to $t \in[0, \tau)$ in $X$ and

$$
\frac{d}{d t} \widetilde{S}_{B}(t) x=S(t) x+B \widetilde{S}_{B}(t) x+\int_{0}^{t} S^{(n+1)}(t-s) B \widetilde{S}_{B}(s) x d s .
$$

Define $S_{B}(t) x:=\frac{d}{d t} \widetilde{S}_{B}(t) x$ for $x \in X$. Then $S_{B}(t)$ satisfies (3.8) and $\left\{S_{B}(t)\right\}_{t \in[0, \tau)}$ is the $n$-times integrated semigroup generated by $A+B$.

We note that in Theorems 3.1 and 3.2 , since both $A$ and $A+B$ are closed, from the calculation

$$
\begin{aligned}
\|x\|_{[D(A+B)]} & =\|x\|+\|(A+B) x\| \leq\|x\|+\|A x\|+\|B x\|_{[D(A)]} \\
& \leq\left(1+\|B\|_{[D(A)]}\right)\|x\|_{[D(A)]} \quad \forall x \in D(A),
\end{aligned}
$$

it follows that the graph norms $\|\cdot\|_{[D(A)]}$ and $\|\cdot\|_{[D(A+B)]}$ on $D(A)$ are equivalent. Therefore if $B \in L([D(A)])$ then $B \in L([D(A+B)])$ and vice versa, hence it is routine to show that if $\operatorname{Im}(B) \subseteq D\left(A^{n+1}\right)$ and $A+B$ generates an $n$-times integrated semigroup then so does $A$.

4. Local complete second order differential equations. Consider the following local complete second order abstract differential equation (see 
Section 1):

$$
\begin{aligned}
& u^{\prime \prime}(t)=A u(t)+B u^{\prime}(t) x, \quad t \in[0, \tau) ; \\
& u(0)=x, \quad u^{\prime}(0)=y, \quad x, y \in X,
\end{aligned}
$$

where $0<\tau \leq \infty$ and $A, B$ are closed on $X$ throughout, but sometimes $B$ is bounded.

In this section we will apply the theorems produced in Sections 2 and 3 to establish several sufficient conditions for the existence and uniqueness of strong solutions of (1.1) (see Definition 4.1) and several equivalent conditions for the existence and uniqueness of integrated mild solutions of (1.1) (see Definition 4.2).

Definition 4.1. A function $u(\cdot, x, y):[0, \tau) \rightarrow X$ is a strong solution of $(1.1)$ if $u(\cdot, x, y) \in C^{2}([0, \tau), X) \cap C([0, \tau),[D(A)]), B u^{\prime}(\cdot, x, y) \in C([0, \tau), X)$ and $u(\cdot, x, y)$ satisfies (1.1).

When $B$ is bounded on $X$, define

$$
\mathcal{N}:=\left(\begin{array}{cc}
0 & I \\
A & B
\end{array}\right) .
$$

Then it is easy to see that $\mathcal{N}$ with domain $D(A) \times X$ is a closed linear operator on $X \times X$, endowed with the norm $\left\|\left(\begin{array}{l}x \\ y\end{array}\right)\right\|:=\|x\|+\|y\|$, and we may reduce (1.1) to the following local first order abstract differential equation on $X \times X$ (see [10]):

$$
w^{\prime}(t)=\mathcal{N} w(t), \quad w(0)=\left(\begin{array}{l}
x \\
y
\end{array}\right), \quad t \in[0, \tau), x, y \in X .
$$

Definition 4.2. Assume $B$ is bounded and $x, y \in X$ are given. A function $v(\cdot, x, y):[0, \tau) \rightarrow X$ is an n-times integrated mild solution of (1.1) if $v(0, x, y)=0, v(\cdot, x, y) \in C([0, \tau), X), \int_{0}^{t}(t-s) v(s, x, y) d s \in D(A)$ for $t \in[0, \tau)$ and

$$
\begin{aligned}
v(t, x, y)= & A \int_{0}^{t}(t-s) v(s, x, y) d s+B \int_{0}^{t} v(s, x, y) d s \\
& -\frac{t^{n+1}}{(n+1) !} B x+\frac{t^{n}}{n !} x+\frac{t^{n+1}}{(n+1) !} y, \quad x, y \in X .
\end{aligned}
$$

If $B=0$ then (4.2) reduces to (1.5).

The following theorem is a slight generalization of [1, Theorem 3.14.7].

TheOrem 4.3. The following conditions (i)-(iii) are equivalent:

(i) (1.4) has a unique $n$-times integrated mild solution for every pair $x, y \in X$.

(ii) A generates an $n$-times integrated cosine family on $X$. 
(iii) $\mathcal{A}=\left(\begin{array}{ll}0 & I \\ A & 0\end{array}\right)$ generates an $(n+1)$-times integrated semigroup on $X \times X$.

If $B$ is bounded and $\operatorname{Im}(B) \subseteq D\left(A^{[(n+1) / 2]}\right)$ then (i)-(iii) are equivalent to the following two statements:

(iv) $\mathcal{N}$ generates an $(n+1)$-times integrated semigroup on $X \times X$.

(v) (1.1) has a unique $n$-times integrated mild solution for every pair $x, y \in X$.

If the equivalent conditions (iv) and (v) hold, then

(vi) (1.1) has a unique strong solution for $(x, y) \in D\left(A^{1+[(n+1) / 2]}\right) \times$ $D\left(A^{1+[n / 2]}\right)$.

Proof. The equivalence of (i) and (ii) was proved in Theorem 1.7.

(i) $\Leftrightarrow$ (iii). Consider the following problem:

$$
\left(\begin{array}{l}
w_{1}^{\prime}(t) \\
w_{2}^{\prime}(t)
\end{array}\right)=\mathcal{A}\left(\begin{array}{l}
w_{1}(0) \\
w_{2}(0)
\end{array}\right)=\left(\begin{array}{l}
x \\
y
\end{array}\right) .
$$

If $v(\cdot, x, y)$ is an $n$-times integrated mild solution of (1.4) then

$$
\left(\begin{array}{c}
\int_{0}^{t} v(s, x, y) d s \\
v(t, x, y)-\frac{t^{n}}{n !} x
\end{array}\right)
$$

is an $(n+1)$-times integrated mild solution of (4.3). In fact, from

$$
v(t, x, y)=A \int_{0}^{t}(t-s) v(s, x, y) d s+\frac{t^{n}}{n !} x+\frac{t^{n+1}}{(n+1) !} y,
$$

it follows that

$$
\left(\begin{array}{c}
\int_{0}^{t} v(s, x, y) d s \\
v(t, x, y)-\frac{t^{n}}{n !} x
\end{array}\right)=\mathcal{A} \int_{0}^{t}\left(\begin{array}{c}
\int_{0}^{s} v(r, x, y) d r \\
v(s, x, y)-\frac{s^{n}}{n !} x
\end{array}\right) d s+\frac{t^{n+1}}{(n+1) !}\left(\begin{array}{l}
x \\
y
\end{array}\right) .
$$

Conversely, assume that $w(\cdot, x, y)=\left(\begin{array}{c}w_{1}(\cdot, x, y) \\ w_{2}(\cdot, x, y)\end{array}\right)$ is an $(n+1)$-times integrated mild solution of (4.3):

$$
\left(\begin{array}{l}
w_{1}(t, x, y) \\
w_{2}(t, x, y)
\end{array}\right)=\mathcal{A} \int_{0}^{t}\left(\begin{array}{l}
w_{1}(s, x, y) \\
w_{2}(s, x, y)
\end{array}\right) d s+\frac{t^{n+1}}{(n+1) !}\left(\begin{array}{l}
x \\
y
\end{array}\right) .
$$

Then

$$
\begin{aligned}
& w_{1}(t, x, y)=\int_{0}^{t} w_{2}(s, x, y) d s+\frac{t^{n+1}}{(n+1) !} x, \\
& w_{2}(t, x, y)=A \int_{0}^{t} w_{1}(s, x, y) d s+\frac{t^{n+1}}{(n+1) !} y .
\end{aligned}
$$


(4.4) implies that $w_{1}(t, x, y)$ is continuously differentiable with respect to $t \in[0, \tau)$. Set $v(t, x, y)=w_{1}^{\prime}(t, x, y)=w_{2}(t, x, y)+\frac{t^{n}}{n !} x$. Then (4.4) and (4.5) imply that $v(\cdot, x, y)$ is an $n$-times integrated mild solution of (1.4). An application of Theorem 1.3 shows that (i) and (iii) are equivalent. Thus we have proved that (i), (ii) and (iii) are equivalent.

(iii) $\Leftrightarrow\left(\right.$ iv) . Define $\mathcal{B}:=\left(\begin{array}{ll}0 & 0 \\ 0 & B\end{array}\right)$. Then the condition $\operatorname{Im}(B) \subseteq D\left(A^{[(n+1) / 2]}\right)$, together with the relations (see $[3,17]$ )

$$
\mathcal{A}^{2 k}=\left(\begin{array}{cc}
A^{k} & 0 \\
0 & A^{k}
\end{array}\right), \quad \mathcal{A}^{2 k+1}=\left(\begin{array}{cc}
0 & A^{k} \\
A^{k+1} & 0
\end{array}\right) \quad \forall k \in \mathbb{N} \cup\{0\},
$$

implies that $\operatorname{Im}(\mathcal{B}) \subseteq D\left(\mathcal{A}^{n+1}\right)$. An application of Theorem 2.5 and the remark following Corollary 2.6 show that (iii) and (iv) are equivalent.

(iv) $\Leftrightarrow(\mathrm{v})$. Assume (iv) is true. From Theorem 1.3, for every $\left(\begin{array}{l}x \\ y\end{array}\right) \in X \times X$ there exists a unique continuous function $\left(\begin{array}{c}w_{1}(\cdot, x, y) \\ w_{2}(\cdot, x, y)\end{array}\right):[0, \tau) \rightarrow X \times X$ such that $w_{1}(0, x, y)=0=w_{2}(0, x, y)$ and

$$
\left(\begin{array}{l}
w_{1}(s, x, y) \\
w_{2}(t, x, y)
\end{array}\right)=\left(\begin{array}{cc}
0 & I \\
A & B
\end{array}\right)\left(\begin{array}{l}
\int_{0}^{t} w_{1}(s, x, y) d s \\
\int_{0}^{t} w_{2}(s, x, y) d s
\end{array}\right)+\frac{t^{n+1}}{(n+1) !}\left(\begin{array}{l}
x \\
y
\end{array}\right) \quad \forall t \in[0, \tau) .
$$

The last relation is equivalent to

$$
\begin{aligned}
& w_{1}(s, x, y)=\int_{0}^{t} w_{2}(s, x, y) d s+\frac{t^{n+1}}{(n+1) !} x, \\
& w_{2}(s, x, y)=A \int_{0}^{t} w_{1}(s, x, y) d s+B \int_{0}^{t} w_{2}(s, x, y) d s+\frac{t^{n+1}}{(n+1) !} y .
\end{aligned}
$$

(4.7) implies that $w_{1}(\cdot, x, y)$ is continuously differentiable on $[0, \tau)$. Define $v(t, x, y)=w_{1}^{\prime}(t, x, y)=w_{2}(t, x, y)+\frac{t^{n}}{n !} x$. Then $v(0, x, y)=0$ and (4.7), (4.8) give

$$
\begin{aligned}
v(t, x, y)= & A \int_{0}^{t}(t-s) v(s, x, y) d s \\
& +B \int_{0}^{t} v(s, x, y) d s-\frac{t^{n+1}}{(n+1) !} B x+\frac{t^{n}}{n !} x+\frac{t^{n+1}}{(n+1) !} y
\end{aligned}
$$

that is, $v(\cdot, x, y)$ is an $n$-times integrated mild solution of (1.1) by Definition 4.2. From the uniqueness of the solutions of (1.1) we may show that $v(\cdot, x, y)$ is uniquely determined by $x, y \in X$. Thus (v) is true.

Conversely, assume that (1.1) has a unique $n$-times integrated mild solution $v(\cdot, x, y)$ for $x, y \in X$. Then $\left(\begin{array}{c}\int_{0}^{t} v(s, x, y) d s \\ v(t, x, y)-\frac{t^{n}}{n !} x\end{array}\right)$ is the unique $(n+1)$ times integrated mild solution of (4.1). Theorem 1.3 implies that (iv) is true. 
To prove (vi), from $\mathcal{N}=\mathcal{A}+\mathcal{B}$ and $\operatorname{Im}(B) \subseteq D\left(A^{[(n+1) / 2]}\right)$, we can immediately show that $D\left(\mathcal{N}^{n+2}\right)=D\left(A^{1+[(n+1) / 2]}\right) \times D\left(A^{1+[n / 2]}\right)$ (see (iii) $\Leftrightarrow$ (iv) and $[3,17])$. Thus (vi) is a direct consequence of (iv) of Theorem 1.3.

It is worthwhile to mention that the condition $\operatorname{Im}(B) \subseteq D\left(A^{[n+1) / 2]}\right)$ is not necessary for the equivalence of (iv) and (v) in Theorem 4.3.

Corollary 4.4. Assume $\tau=\infty$ and $w_{0}>0$. Then the following conditions (i)-(iii) are equivalent:

(i) (1.4) has a unique $n$-times integrated mild solution $u(\cdot, x, y)$ satisfying

$$
\|u(t, x, y)\|=O\left(e^{\omega_{0} t}\right)
$$

for every pair $x, y \in X$.

(ii) A generates an n-times integrated cosine family $\{\mathcal{C}(t)\}_{t \geq 0}$ satisfying

$$
\|\mathcal{C}(t)\|=O\left(e^{\omega_{0} t}\right) .
$$

(iii) $\mathcal{A}$ generates an $(n+1)$-times integrated semigroup $\{\mathcal{S}(t)\}_{t \geq 0}$ satisfying

$$
\|\mathcal{S}(t)\|=O\left(e^{\omega_{0} t}\right)
$$

If the equivalent conditions (i)-(iii) hold, and $B$ is bounded with $\operatorname{Im}(B)$ $\subseteq D\left(A^{[(n+1) / 2]}\right)$, then

(iv) $\mathcal{N}$ generates an $(n+1)$-times integrated semigroup $\{\widetilde{\mathcal{S}}(t)\}_{t \geq 0}$ on $X \times X$ satisfying

$$
\|\widetilde{\mathcal{S}}(t)\|=O\left(e^{\left(\omega_{0}+\|B\|\right) t}\right) .
$$

Corollary $4.5([3,16])$. Assume $\tau=\infty$ and $\omega_{0}>0$. Then the following conditions (i)-(iii) are equivalent.

(i) A generates an $n$-times integrated cosine family $\{\mathcal{C}(t)\}_{t \geq 0}$ satisfying

$$
\lim _{h \rightarrow 0^{+}} \frac{1}{h}\|\mathcal{C}(t+h)-\mathcal{C}(t)\| \leq M_{0} e^{\omega_{0} t}
$$

where $M_{0}>0$ is a constant.

(ii) $\left(\omega_{0}^{2}, \infty\right) \subseteq \varrho(A)$, and

$$
\left\|\left(\lambda-\omega_{0}\right)^{k+1}\left[\lambda^{2-n} R\left(\lambda^{2}, A\right)\right]^{(k)}\right\| \leq M_{0} k ! \quad \forall k \in \mathbb{N} \cup\{0\} .
$$

(iii) $\mathcal{A}$ generates an $(n+1)$-times integrated semigroup $\{\mathcal{S}(t)\}_{t \geq 0}$ satisfying

$$
\lim _{h \rightarrow 0^{+}} \frac{1}{h}\|\mathcal{S}(t+h)-\mathcal{S}(t)\| \leq M_{1} e^{\omega_{0} t} .
$$

If the equivalent conditions (i)-(iii) hold, and $B$ is bounded with $\operatorname{Im}(B) \subseteq$ $D\left(A^{[(n+1) / 2]}\right)$, then 
(iv) $\mathcal{N}$ generates an $(n+1)$-times integrated semigroup $\{\widetilde{S}(t)\}_{t \geq 0}$ satisfying

$$
\lim _{h \rightarrow 0^{+}} \frac{1}{h}\|\widetilde{\mathcal{S}}(t+h)-\widetilde{\mathcal{S}}(t)\| \leq M_{2} e^{\left(\omega_{0}+\|B\|\right) t} .
$$

In (iii) and (iv), $M_{1}, M_{2}>0$ are also constants.

Our Theorem 4.3 and its corollaries improve [10, Theorems 4.1 and 4.7]. If closed linear operators $A$ and $B$ are both unbounded then we reduce (1.1) to (see [10])

$$
\begin{aligned}
& w^{\prime}(t)=\mathcal{M} w(t), \quad \mathcal{M}=\left(\begin{array}{ll}
B & I \\
A & 0
\end{array}\right), t \in[0, \tau) ; \\
& w(0)=\left(\begin{array}{c}
x \\
y-B x
\end{array}\right), \quad x, y \in X .
\end{aligned}
$$

The operator $\mathcal{M}$ with domain $(D(A) \cap D(B)) \times X$ is closed on $X \times X$.

Theorem 4.6. Assume $B \in L([D(A)])$ and $\operatorname{Im}(B) \subseteq D\left(A^{[(n+1) / 2]}\right)$. Then the conditions (i), (ii) and (iii) in Theorem 4.3 are equivalent to the following:

$\left(i v^{\prime}\right) \mathcal{M}$ generates an $(n+1)$-times integrated semigroup. If $\left(\mathrm{iv}^{\prime}\right)$ holds, then

$\left(\mathrm{v}^{\prime}\right)(1.1)$ has a unique strong solution for all $(x, y) \in D\left(A^{1+[(n+1) / 2]}\right) \times$ $D\left(A^{1+[n / 2]}\right)$.

Proof. (iii) of Theorem $4.3 \Leftrightarrow\left(\mathrm{iv}^{\prime}\right)$. Recall that $\mathcal{A}=\left(\begin{array}{cc}0 & I \\ A & 0\end{array}\right)$ and $\mathcal{B}=$ $\left(\begin{array}{ll}0 & 0 \\ 0 & B\end{array}\right)$. Then $\mathcal{B} \in L([D(\mathcal{A})])$ and $\|\mathcal{B}\|_{[D(\mathcal{A})]} \leq\|B\|_{[D(A)]}$. From the equivalence of (iii) and (iv) in Theorem 4.3 under the condition on $B$, we have $\operatorname{Im}(\mathcal{B}) \subseteq D\left(\mathcal{A}^{n+1}\right)$. Thus (iii) and $\left(\mathrm{iv}^{\prime}\right)$ are equivalent by Theorem 3.2 and the remark following it.

$\left(\mathrm{v}^{\prime}\right)$ can be proved in the same way as (vi) of Theorem 4.3.

There are also two corollaries of Theorem 4.6 similar to Corollaries 4.4 and 4.5. We leave the details to the reader.

To end this section, we consider two examples.

Following [8, Chapter VIII], let $X=l^{2}$ be the space of all sequences $x=\left\{\xi_{1}, \xi_{2}, \ldots\right\}$ satisfying $\|x\|^{2}=\sum_{k=0}^{\infty}\left|\xi_{k}\right|^{2}<\infty$ and let $A, B$ be the operators

$$
A\left\{\xi_{k}\right\}:=\left\{a_{k} \xi_{k}\right\}, \quad B\left\{\xi_{k}\right\}=\left\{b_{k} \xi_{k}\right\},
$$

where $\left\{a_{k}\right\},\left\{b_{k}\right\}$ are sequences of complex numbers. It is easy to see that $A$ and $B$ are closed and densely defined. 
Example 4.7. From [8, Section VIII.2, Example 2.1], for any function $\Omega(\cdot)$ on $[0, \infty)$, bounded on every compact subset, there exist $A, B$ as in (4.10) such that $(1.1)$ has a unique strong solution $u(\cdot, x, y)$ satisfying $u(0, x, y)=x, u^{\prime}(0, x, y)=y$ for $(x, y)$ in a dense subset of $X \times X$ and

$$
\|u(t)\| \geq \Omega(t)\|x\|, \quad\left\|u^{\prime}(t)\right\| \geq \Omega(t)\|y\| .
$$

This implies that (1.1) has solutions which are not exponentially bounded.

In the following example, we assume that $X=c_{0}$, the space of all sequences $x=\left\{\xi_{k}\right\}$ satisfying $\lim _{k \rightarrow \infty} \xi_{k}=0$. Endowed with the sup norm, $X$ is a Banach space.

Example 4.8. Assume $0<\tau<\infty$ and $A=0$ in (1.1). Then there exists $B$ as in (4.10) such that equation (1.1) has a unique strong solution $u(0)=0, u^{\prime}(0)=y$ for $y$ in a dense subset of $X$, but (1.1) with the given initial data has no strong solution on the interval $\left[0, \tau^{\prime}\right)$ for any $\tau^{\prime}>\tau$.

Proof. From the given initial data, equation (1.1) is equivalent to

$$
u_{k}^{\prime \prime}(t)=b_{k} u_{k}^{\prime}(t), \quad t \in[0, \tau) ; \quad u_{k}(0)=0, \quad u_{k}^{\prime}(0)=\eta_{k} \quad \forall k \in \mathbb{N},
$$

where $b_{k}$ is the $k$ th entry of $B$ in (4.10) and $y:=\left\{\eta_{k}\right\} \in X$. Without loss of generality we may assume $0 \neq\left|\eta_{k}\right|<1$ for all $k \in \mathbb{N}$. The roots of the characteristic equation of (4.11) are $\lambda_{0}=0, \lambda_{k}=b_{k}$ and the solution of (4.11) is

$$
u_{k}(t)=\frac{\eta_{k}}{\lambda_{k}}\left(e^{\lambda_{k} t}-1\right) \quad \forall k \in \mathbb{N} .
$$

Hence $u(\cdot):=\left\{u_{k}(t)\right\}$ is the unique strong solution of (1.1) with $A=0$ and the initial data $u(0)=0, u^{\prime}(0)=y$.

We now show that $u(\cdot)$ cannot be extended to $\left[0, \tau^{\prime}\right)$ for any $\tau^{\prime}>\tau$. Define $\zeta_{k}:=1 /\left|\eta_{k}\right|$; then $\zeta_{k}>1$ and $\lim _{k \rightarrow \infty} \zeta_{k}=\infty$. Choose

$$
\lambda_{k}:=\frac{1}{\tau} \log \zeta_{k}+\frac{i}{\tau}\left[\zeta_{k}^{2 / \sqrt{\zeta_{k}}}-\left(\log \zeta_{k}\right)^{2}\right]^{1 / 2} \quad \forall k \in \mathbb{N} .
$$

This implies

$$
\begin{aligned}
\left|u_{k}(t)\right| & \geq \frac{\left|e^{\lambda_{k} t}\right|-1}{\zeta_{k}\left|\lambda_{k}\right|}=\frac{e^{(t / \tau) \log \zeta_{k}}}{\zeta_{k}\left|\lambda_{k}\right|}-\frac{1}{\zeta_{k}\left|\lambda_{k}\right|} \\
& =\left(\tau \zeta_{k}^{t / \tau}\right) /\left(\zeta_{k}^{1+1 / \sqrt{\log \zeta_{k}}}\right)-\tau /\left(\zeta_{k}^{1+1 / \sqrt{\log \zeta_{k}}}\right) \\
& =\tau \zeta_{k}^{t / \tau-1-1 / \sqrt{\log \zeta_{k}}}-\tau /\left(\zeta_{k}^{1+1 / \sqrt{\log \zeta_{k}}}\right) .
\end{aligned}
$$

If we note that

$$
\sqrt{\log \zeta_{k}} \rightarrow \infty \quad \text { and } \quad \zeta_{k}^{1+1 / \sqrt{\log \zeta_{k}}} \rightarrow \infty
$$


then, for fixed $t>\tau,(4.12)$ implies that $u_{k}(t) \rightarrow \infty$ as $k \rightarrow \infty$. Hence $\left\{u_{k}(t)\right\}$ is not in $X$ whenever $t>\tau$, that is, as an $X$-valued function of $t$, it cannot be extended to the interval $\left[0, \tau^{\prime}\right)$ for any $\tau^{\prime}>\tau$, or equivalently, (1.1) has no strong solution with $[0, \tau)$ replaced by $\left[0, \tau^{\prime}\right)$.

Acknowledgments. The authors highly appreciate the referee's indicating reference [1] and indicating that our Theorem 4.3 is in connection with Theorem 3.14 .7 of $[1]$.

\section{References}

[1] W. Arendt, Ch. J. K. Batty, M. Hieber and F. Neubrander, Vector-Valued Laplace Transforms and Cauchy Problems, Birkhäuser, 2001.

[2] W. Arendt, O. El-Mennaoui and V. Keyantuo, Local integrated semigroups: evolution with jumps of regularity, J. Math. Anal. Appl. 186 (1994), 572-595.

[3] W. Arendt and H. Kellermann, Integrated solutions of Volterra integrodifferential equations and applications, in: Pitman Res. Notes Math. Ser. 190, Longman, 1989, $21-51$.

[4] R. deLaubenfels, Existence Families, Functional Calculi and Evolution Equations, Lecture Notes in Math. 1570, Springer, 1994.

[5] R. deLaubenfels, C. Sun and S. W. Wang, Regularized semigroups, existence families and the abstract Cauchy problem, Differential Integral Equations 8 (1995), 14771496.

[6] W. Desch and W. Schappacher, On relatively bounded perturbations of linear $C_{0}$ semigroups, Ann. Scuola Norm. Sup. Pisa Cl. Sci. 11 (1984), 327-341.

[7] K. J. Engel and R. Nagel, One-Parameter Semigroups for Linear Evolution Equations, Grad. Texts in Math. 194, Springer, New York, 1999.

[8] H. O. Fattorini, Second Order Linear Diferential Equations in Banach Spaces, North-Holland Math. Stud. 108, North-Holland, 1985.

[9] J. A. Goldstein, Semigroups of Linear Operators and Applications, Oxford Univ. Press, 1985.

[10] F. Neubrander, Integrated semigroups and their applications to complete second order Cauchy problems, Semigroup Forum 38 (1989), 233-251.

[11] - Integrated semgroups and their applications to the abstract Cauchy problem, Pacific J. Math. 135 (1988), 111-157.

[12] A. Pazy, Semigroups of Linear Operators and Applications to Partial Differential Equations, Springer, New York, 1983.

[13] J. E. Shi and W. X. Zheng, On local integrated cosine functions, Northeast Math. J. 17 (2001), 21-26.

[14] H. Wang and S. W. Wang, C-cosine operator functions and their applications to the second order abstract Cauchy problem, in: Functional Analysis in China, Kluwer, 1996, 333-350.

[15] S. W. Wang and Z. Huang, Strongly continuous integrated C-cosine operator functions, Studia Math. 126 (1997), 273-289. 
[16] Q. Zheng, Perturbations and applications of integrated semigroups, Acta Math. Sinica 9 (1993), 252-260 (in Chinese).

[17] - Integrated cosine functions and second order Cauchy problems, preprint.

Department of Applied Mathematics

Mailing address:

Nanjing Audit Institute

Nanjing, Jiangsu 210029

Sheng Wang Wang

P.R. China

Department of Mathematics

Nanjing University

Nanjing, Jiangsu 210093

P.R. China

E-mail: wang2598@nju.edu.cn

Received November 25, 2003

Revised version January 24, 2005 